\title{
Salt Tolerance Enhancement of Liquid Chromatography- Matrix-Assisted Laser Desorption/Ionization-Mass Spectrometry Using Matrix Additive Methylenediphosphonic Acid
}

\author{
Yuki Ohta, Shinichi Iwamoto, ${ }^{*}$ Shin-ichirou Kawabata, \\ Ritsuko Tanimura, and Koichi Tanaka \\ Koichi Tanaka Laboratory of Advanced Science and Technology, Shimadzu Corporation, Nakagyo-ku, Kyoto 604-8511, Japan
}

\begin{abstract}
Mass spectrometry (MS) is a highly sensitive analytical technique that is often coupled with liquid chromatography (LC). However, some buffering salts used in LC (e.g., phosphate and tris(hydroxymethyl) aminomethane (Tris)) are incompatible with MS since they cause ion-source contamination and signal suppression. In this study, we examined salt tolerance of MALDI and applied a matrix additive methylenediphosphonic acid (MDPNA) to reduce salt-induced signal suppression. MDPNA significantly improved the salt tolerance of MALDI-MS. Using ammonium formate buffer at $\mathrm{pH}$ 5.0, the effective range of buffering salt concentration in MALDI-MS using MDPNA was estimated up to $250 \mathrm{mM}$. MDPNA reduced signal suppression caused by buffering salts at $\mathrm{pH} 4.0$ to 8.0. We observed that MDPNA effectively worked over a wide range of buffer conditions. MDPNA was further applied to hydrophilic interaction chromatography (HILIC) and chromatofocusing-MALDI-MS. As a result, the analytes in the eluent containing high-concentration salts were detected with high sensitivity. Thus, our study provides simple and fast LC-MALDIMS analysis technique not having strict limitation of buffering condition in LC by using matrix additive MDPNA.

Please cite this article as: Y. Ohta, et al., Salt Tolerance Enhancement of Liquid Chromatography-MatrixAssisted Laser Desorption/Ionization-Mass Spectrometry Using Matrix Additive Methylenediphosphonic Acid, Mass Spectrom (Tokyo) 2014; 3(1): A0031; DOI: 10.5702/massspectrometry.A0031
\end{abstract}

Keywords: matrix-assisted laser desorption/ionization, matrix additive, liquid chromatography, chromatofocusing, hydrophilic interaction chromatography

(Received May 7, 2014; Accepted July 26, 2014)

\section{INTRODUCTION}

Buffering agents are commonly used to achieve good separation, high resolution, and high reproducibility in liquid chromatography (LC). ${ }^{1-3)}$ Mass spectrometry (MS) is a highly selective and sensitive analytical technique that is important for LC analysis, ${ }^{4,5)}$ thus the importance of LC-MS instrument is increasing. However, almost all ionization methods (e.g., electrospray ionization (ESI), matrix-assisted laser desorption/ionization (MALDI), and atmospheric pressure chemical ionization (APCI)) used for MS are incompatible with some buffering salts commonly used for LC (e.g., phosphate, citrate, and Tris), because they often contaminate the ionization source of mass spectrometer and decrease the ion signal due to buffer-induced ionization suppression. $^{6,7)}$

There are many LC separation modes applied to the frontend of MS. For example, chromatofocusing is one of useful separation modes that allows separation of proteins based on their isoelectric point $(\mathrm{p} I){ }^{8)}$ Proteins which have different $\mathrm{p} I$ migrate at different speed through the column, and combining this technique with MS can be powerful tool for identifying unknown analytes because it is possible to obtain $\mathrm{p} I$ value and structural information at the same time. Octadecyl silica (ODS) is the most commonly used mode in LC-MS. However, some hydrophilic compounds cannot be retained in ODS. Hydrophilic interaction chromatography (HILIC) has recently become a major separation mode in LC-MS because it can retain and separate hydrophilic compounds that cannot be retained on ODS columns., ${ }^{49-11)}$ In particular, zwitterionic HILIC is a widely accepted separation mode due to its separation capacity. However, it requires millimolar order of buffering salts that can interfere with analyte ionization. ${ }^{11}$

Salt-induced signal suppression is commonly observed in MALDI-MS like the other ionization methods. However, MALDI may be able to overcome signal suppression. Smirnov et al. (2004) reported that adding ammonium monobasic phosphate to $\alpha$-cyano-4-hydroxycinnamic acid (CHCA), followed by washing the matrix crystal with am-

*Correspondence to: Shinichi Iwamoto, Koichi Tanaka Laboratory of Advanced Science and Technology, Shimadzu Corporation, Nakagyo-ku, Kyoto 604-8511, Japan, e-mail: iwamoto@shimadzu.co.jp 
monium monobasic phosphate water, significantly reduced the matrix adducts and improved peptide detection in MALDI time-of-flight MS. ${ }^{12)}$ Asanuma et al. (2009) reported that the ionization efficiency of proteins in a sodium dodecyl sulfate solution was improved by adding a strong anionexchange silica gel to the matrix solution. ${ }^{13)}$ Kuyama et al. (2008) reported that adding the matrix additive methylenediphosphonic acid (MDPNA) to the matrix 2,5-dihydroxybenzoic acid (DHB) improved the detection of phosphopeptides and peptides in tris(hydroxymethyl)aminomethane (Tris) hydrochloride buffers without desalting. ${ }^{14)}$ Especially, MDPNA is water soluble and easy to handle, and can be applied simply by adding it to matrixes. If MDPNA is effective against various buffers commonly used in LC, MDPNA is an ideal matrix additive for LC-MALDI-MS.

This study aims to reinforce the salt resistance of MALDIMS and widen the range of separation-mode options in LCMALDI-MS by using matrix additives. Here, we report on organic and inorganic salt-resistant offline LC-MALDI-MS analysis using MDPNA as a matrix additive.

\section{EXPERIMENTAL}

\section{Materials}

The peptides angiotensin I (Ang I, purity $\geq 90 \%$ ), angiotensin II (Ang II, $\geq 93 \%$ ), glu-1-fibrinopeptide B ([Glu1]Fib, $\geq 90 \%), N$-acetyl-renin substrate tetradecapeptide porcine ( $N$-acetyl-renin, $\geq 97 \%$ ), adrenocorticotropic hormone (ACTH) fragment 1-17 (ACTH 1-17, 297\%), ACTH fragment 18-39 (ACTH 18-39, $\geq 97 \%$ ), and ACTH fragment 7-38 (ACTH 7-38, $\geq 97 \%$ ) were purchased from SigmaAldrich (St. Louis, MO). CHCA and DHB matrixes were purchased from LaserBio Labs (Sophia-Antipolis Cedex, France). Tryptic protein digestion standards of yeast enolase (ENO, SwissProt P00924), phosphorylase b (PhosB, SwissProt P00489), alcohol dehydrogenase (ADH, SwissProt P00330), and bovine serum albumin (BSA, SwissProt P02769) were purchased from Waters (MassPREP, $\approx 1 \mathrm{nmol} /$ vial; Milford, MA). Ethane-1-hydroxy-1,1-diphosphonic acid (HEDP) and 1,2-ethanediphosphonic acid (EDPNA) were purchased from Alfa Aesar (Lancashire, UK). MDPNA was purchased from Tokyo Chemical Industry Co., Ltd. (Tokyo, Japan). Phosphonoacetic acid (PAC) and phosphoric acid ( $\mathrm{PA}, \geq 85 \mathrm{wt} \%$ in $\mathrm{H}_{2} \mathrm{O}$ ) were purchased from Sigma (St. Louis, MO). Tris was purchased from Bio-Rad (Hercules, CA). Bis(2-hydroxyethyl)-amino-tris(hydroxymethyl)-methane (Bis-Tris), piperazine, imidazole, 1-methylpiperazine, diethanolamine (DEA), and ammonium formate were purchased from Sigma-Aldrich and were of the highest grade available. Milli-Q (Merck Millipore, Billerica, MA) water was used throughout the experiment. The other chemicals were purchased from Wako Pure Chemical Industries, Ltd. (Osaka, Japan) and were of the highest grade.

\section{Stock solutions and matrixes}

A peptide mixture $(6 \mathrm{pmol} / \mu \mathrm{L}$ of Ang I, Ang II, [Glu1]Fib, N-acetyl-renin, ACTH 1-17, and ACTH 18-39 and $4 \mathrm{pmol} / \mu \mathrm{L}$ of ACTH $7-38$ in $0.1 \%$ TFA/water) was prepared. We refer to the peptide mixture as the seven-peptide mix. It was divided into small aliquots $(200 \mu \mathrm{L})$ and stored at $-20^{\circ} \mathrm{C}$ until use. The protein digestion standards (ENO, $\mathrm{Phos}, \mathrm{ADH}$, and $\mathrm{BSA}$ ) in vials were dissolved in $0.1 \mathrm{~mL}$ of
$0.1 \%$ TFA/water, and the solutions were dispensed in $100 \mu \mathrm{L}$ aliquots in $0.5 \mathrm{~mL}$ Eppendorf tubes and stored at $-20^{\circ} \mathrm{C}$ before use (final concentration, $\approx 10 \mathrm{pmol} / \mu \mathrm{L}$ each). Aliquots of MDPNA, EDPNA, PAC, and HEDP were dissolved in Milli-Q water (final concentration, $1 \% \mathrm{w} / \mathrm{v}$ each), and the resulting solutions were stored at $4^{\circ} \mathrm{C}$ until use. An aliquot of PA solution was diluted with Milli-Q water (final concentration, $1 \% \mathrm{w} / \mathrm{v})$. An aliquot of diammonium citrate (DAC) was dissolved in Milli-Q water (final concentration, $10 \mathrm{mM}$, $\mathrm{pH} 4.8)$. Aliquots of CHCA (5.0 mg) and DHB (5.0 mg) were dissolved in $1.0 \mathrm{~mL}$ of $0.05 \% \mathrm{TFA}$ in $50 \%(\mathrm{v} / \mathrm{v})$ acetonitrile $(\mathrm{MeCN}) /$ water; these solutions were stored in the dark and used within three days of preparation. In addition, $5 \mathrm{mM}$ each of Tris, Bis-Tris, piperazine, imidazole, 1-methylpiperazine, and DEA mixtures were prepared by dissolving in water, and the mixtures were adjusted to $\mathrm{pH} 8.0,6.0$, and 4.0 with concentrated $\mathrm{HCl}$. We refer to the organic buffering salt mixtures as $\mathrm{pH} 8.0,6.0$, and 4.0 amine buffer.

\section{Mass spectrometry}

All MALDI-MS data were acquired by MALDI-TOFMS (AXIMA Performance) or MALDI-QIT-TOFMS (AXIMA Resonance) equipped with a nitrogen UV laser $(337 \mathrm{~nm})$ and processed using LaunchPad with LC-MALDI optional functionality (all instruments and software; Shimadzu/Kratos, Manchester, UK). Base peak chromatograms (BPCs) and extracted ion chromatograms (XICs) were obtained using mass++ (Ver. 2). ${ }^{15)}$ All statistical analyses were conducted with statistical software R version 2.13.0. ${ }^{16)}$

\section{Effect of MDPNA on amine buffer}

The seven-peptide mix was diluted twenty-fold with $\mathrm{pH}$ 8.0, 6.0, or 4.0 amine buffer, or $0.1 \%$ TFA. Aliquots of $0.5 \mu \mathrm{L}$ of the seven-peptide mix in amine buffer ( $\mathrm{pH} 8.0,6.0$, or 4.0) or $0.1 \% \mathrm{TFA}$, and $0.5 \mu \mathrm{L}$ of the matrix solution (CHCA or DHB) were mixed on a MALDI target plate (384-well stainless steel target; Shimadzu Corporation, Kyoto, Japan). Then, aliquots of $0.5 \mu \mathrm{L}$ of additive solution (MDPNA, EDPNA, PAC, and HEDP, PA, DAC, TFA, or Milli-Q water) were mixed on the target plate and analyzed by MALDI-TOFMS after drying. MALDI-TOFMS data were acquired by 500 laser shots for one spectrum in linear positive-ion or negativeion mode. A rectangular serpentine raster $(1300 \times 1300 \mu \mathrm{m})$ was applied during data acquisition.

\section{Effect of MDPNA with different buffer concentra- tions}

The seven-peptide mix was diluted twenty-fold with 15 , 60,250 , or $500 \mathrm{mM}$ ammonium formate buffer at $\mathrm{pH} 5.0$. Aliquots of $0.5 \mu \mathrm{L}$ of the seven-peptide mix in ammonium formate buffers, and $0.5 \mu \mathrm{L}$ of the matrix solution (DHB) were mixed on a MALDI target plate. Then, aliquots of $0.5 \mu \mathrm{L}$ of additive solution (1\% MDPNA) were mixed on the target plate and analyzed by MALDI-TOFMS after drying. MALDI-TOFMS data was acquired by 980 laser shots for one spectrum in linear positive-ion mode. A rectangular serpentine raster $(1300 \times 1300 \mu \mathrm{m})$ was applied during data acquisition.

\section{Reduction in signal suppression of tryptic digests by MDPNA}

The protein digestion standards (ENO, PhosB, ADH, or 
BSA) were diluted with amine buffer ( $\mathrm{pH} 8.0$ or 6.0 ) or $0.1 \%$ TFA to concentrations of $100 \mathrm{fmol} / \mu \mathrm{L}$. Aliquots of $0.5 \mu \mathrm{L}$ of the tryptic digests in amine buffer ( $\mathrm{pH} 8.0$ or 6.0 ) or $0.1 \%$ TFA, and $0.5 \mu \mathrm{L}$ of the matrix solution (DHB) were mixed on a MALDI target plate. Then, aliquots of $0.5 \mu \mathrm{L}$ of additive solution (1\% MDPNA) were mixed on the target and analyzed by MALDI-TOFMS after drying. MALDI-TOFMS data was acquired by 500 laser shots for one spectrum in linear positive-ion mode. A rectangular serpentine raster $(1300 \times 1300 \mu \mathrm{m})$ was applied during data acquisition. The sequence coverage ratio was calculated in peptide mass fingerprinting using the Mascot Search Engine (Matrix Science, Boston, MA).

\section{Nano-LC systems}

A nano-LC system equipped with three nano pumps (LC20ADnano), an autosampler (SIL-30ACMP), a nano-valve (FCV nano), and analysis software (LabSolutions) was used (all instruments, Shimadzu Corporation, Kyoto, Japan). The LC system was equipped with a zwitterionic HILIC column (SeQuant ZIC-HILIC, $100 \mathrm{~mm} \times 0.1 \mathrm{~mm}, 3.5 \mu \mathrm{m}, 20 \AA$ A Merck Millipore, Billerica, MA) or a weak anion-exchange column (DEAE; Inertsil AX $150 \mathrm{~mm} \times 0.5 \mathrm{~mm}, 5 \mu \mathrm{m}$; GL Sciences, Tokyo, Japan) for zwitterionic HILIC or chromatofocusing.

\section{Preparing precoated MALDI target}

DHB-precoated plates with MDPNA were prepared as follows. A portion $(1.0 \mu \mathrm{L})$ of the matrix solution containing $2.5 \mathrm{mg} / \mathrm{mL}$ DHB and $1 \%(\mathrm{w} / \mathrm{v})$ MDPNA in 25\% (v/v) MeCN/ water was placed in each well of the $\mu$ Focus MALDI targets (384-circle stainless-steel plate for Bruker and Shimadzu instruments; Hudson Surface Technology, Fort Lee, NJ) and dried (2.5 $\mu \mathrm{g}$ DHB and $10 \mu \mathrm{g}$ MDPNA/well). DHB-precoated plates without MDPNA were prepared followed by the same procedure using a matrix solution containing $2.5 \mathrm{mg} / \mathrm{mL}$ of DHB in 25\% (v/v) MeCN/water.

\section{HILIC-MALDI-MS}

Ammonium formate $20 \mathrm{mM}$ pH 4.5 in $80 \% \mathrm{v} / \mathrm{v}$ acetonitrile/water (mobile phase A) and $20 \mathrm{mM}$ ammonium formate $\mathrm{pH} 4.5$ in water (mobile phase B) were prepared. An aliquot $(0.1 \mu \mathrm{L})$ of a sample containing $100 \mathrm{fmol} / \mu \mathrm{L}$ of BSA tryptic digest in mobile phase A was injected into the HILIC column. The sample was eluted by a binary gradient (flow rate: $200 \mathrm{~nL} / \mathrm{min}, 0$ to $10 \mathrm{~min}$ : B $0 \%, 10$ to $30 \mathrm{~min}: 0 \%$ to $50 \%$, 30 to $35 \mathrm{~min}$ : $50 \%$ to $95 \%, 35$ to $40 \mathrm{~min}: 95 \%$ ), and the eluted sample was spotted onto the DHB-precoated plates with or without MDPNA using a dedicated spotter for LC-MALDIMS (AccuSpot; Shimadzu Corporation, Kyoto, Japan). The sample was analyzed using MALDI-QIT-TOFMS in positive-ion mode (200 laser shots for one spectrum). A rectangular serpentine raster $(700 \times 700 \mu \mathrm{m})$ was applied during data acquisition. Ions for MS/MS were fragmented by collision-induced dissociation with Argon gas in the ion trap.

\section{Chromatofocusing-MALDI-MS}

A buffer containing $25 \mathrm{mM}$ piperazine and $12.5 \mathrm{mM}$ 1-methylpiperazine was prepared, and $\mathrm{pH}$ was adjusted to 5.0 by concentrated $\mathrm{HCl}$ (initialization buffer). The same mixture was prepared, and $\mathrm{pH}$ was adjusted to 3.0 (elution buffer). An aliquot $(0.1 \mu \mathrm{L})$ of a sample containing $20 \mathrm{fmol} /$ $\mu \mathrm{L}$ [Glu1]-fib and $80 \mathrm{fmol} / \mu \mathrm{L}$ ACTH $18-39$ in the initialization buffer was injected into the DEAE column after initialization. The sample was eluted by the elution buffer at a flow rate of $4.0 \mu \mathrm{L} / \mathrm{min}$, and the eluted sample was spotted on the DHB-precoated plates with or without MDPNA using the AccuSpot. The sample was analyzed using MALDI-QITTOFMS. The time-related $\mathrm{pH}$ changes in the solution eluted from the column were measured using a $\mathrm{pH}$ meter equipped with a microelectrode ( $\mathrm{pH}$ meter model D-52; microelectrode Combination Electrode 6069-10C, HORIBA, Kyoto, Japan) before the experiment. The $\mathrm{pH}$ changes were measured at 2 min intervals.

\section{RESULTS AND DISCUSSION}

\section{Effect of MDPNA on the seven-peptide mix}

First, we examined whether MDPNA can reduce signal suppression by amine buffer compounds in MALDI (positive ion mode). The seven-peptide mix in the amine buffer was measured using the CHCA or DHB matrix with or without MDPNA; the results are summarized in Fig. 1.

The signal-to-noise $(S / N)$ ratios of CHCA without MDPNA (Fig. 1-1-1) were better than those of DHB without MDPNA (Fig. 1-2-1), indicating that signal suppression readily occurred when the DHB matrix was used. Although potassium was not intentionally added to the sample, almost all the peptides were detected as not only $[\mathrm{M}+\mathrm{H}]^{+}$ions but also $[\mathrm{M}+\mathrm{K}]^{+}$ions (Figs. 1-1-1 and 1-2-1). Potassium might be a contaminant from the buffering agents. However, the peptide signal intensities were enhanced when MDPNA was added as a matrix additive for both $\mathrm{CHCA}$ and DHB, and the signal enhancements improved the $S / N$ ratios (Figs. 1-1-2 and 1-2-2). Moreover, very few $[\mathrm{M}+\mathrm{K}]^{+}$ions were observed when MDPNA was added as a matrix additive for both CHCA (Fig. 1-1-2) and DHB (Fig. 1-2-2). A simple mass spectrum without undesirable signals of adduct ions (e.g., potassium and sodium adducts) is suitable for automatic data acquisition in MALDI-MS. MDPNA improves the $S / N$ ratio and reduces the generation of unnecessary adduct ions, possibly making it an ideal matrix additive for LCMALDI-MS. The effect of MDPNA on inorganic salts $(\mathrm{NaCl}$ and $\mathrm{Na}_{2} \mathrm{PO}_{4}$ ) was also examined, and an improved $S / N$ ratio was observed (Figs. S-1 and S-2, supporting information). MDPNA effectively reduces signal suppression by both organic and inorganic salts. Moreover, MDPNA was also significantly effective in negative mode MALDI-TOFMS (Figs. S-3 and S-4, supporting information).

Kuyama et al. (2008) and Kjellstrom et al. (2004) examined several matrix additives having phosphoryl group(s) for the highly sensitive detection of phosphopeptides and they concluded that MDPNA or PA is the best matrix additive for detecting phosphopeptides. ${ }^{14,17)}$ We also examined several candidate phosphoryl compounds (MDPNA, EDPNA, PAC, HEDP, and PA; Fig. S-5, supporting information) to ascertain whether MDPNA is a suitable matrix additive for detecting peptides in the buffer solution (Figs. S-6 and S-7, supporting information). Unlike phosphopeptide detection, MDPNA was not always the best matrix additive for peptide analysis. However, it successfully reduced signal suppression caused by buffering salts. Moreover, MDPNA (common name: medronic acid) is used in nuclear medicine to detect bone abnormalities ${ }^{18)}$ and is readily available with 
1) Matrix: $\mathrm{CHCA}$

1-1) without MDPNA
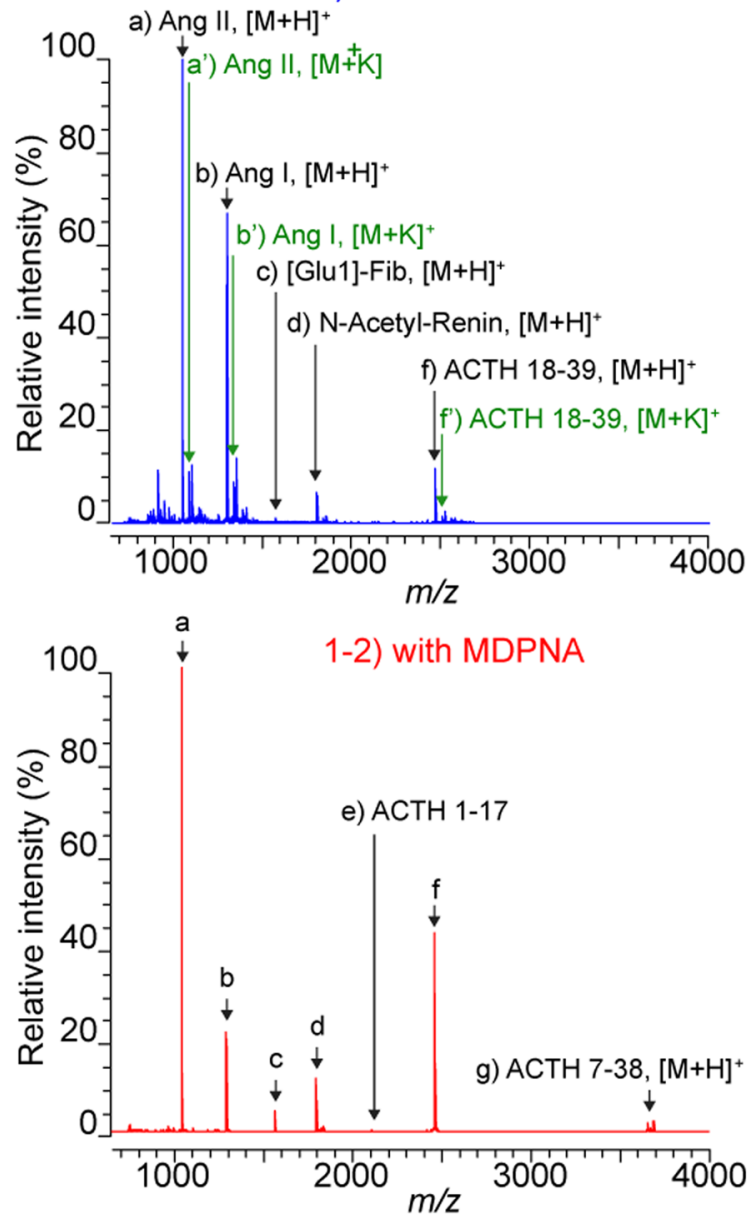

2) Matrix: $D H B$
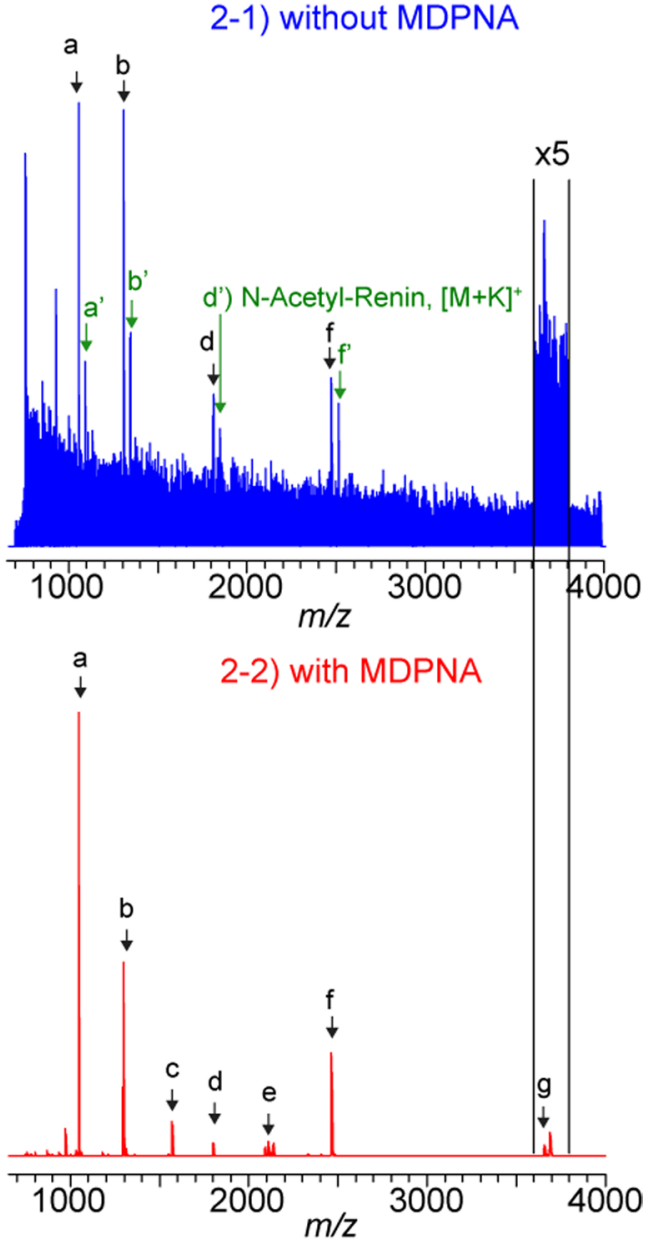

Fig. 1. Mass spectra of the seven-peptide mix in pH 8.0 amine buffer. (1) CHCA and (2) DHB matrixes were used without and with MDPNA.

high purity. Therefore, we used MDPNA as a matrix additive in subsequent studies. DHB and CHCA are commonly used matrixes for peptide analysis in MALDI-MS. Signal suppression induced by buffering salt was more serious when using DHB instead of CHCA as a matrix. However, MDPNA improved the mass spectra acquired using DHB. In other words, MDPNA was more powerful for DHB than for CHCA. DHB is a "cool" matrix. It can reduce undesired fragmentation of fragile analytes and is useful for measuring glycopeptides ${ }^{19)}$ and phosphopeptides. ${ }^{17)}$ The following sections discuss the effects of matrix additives on the DHB.

\section{Usable concentration range of buffering salt in MALDI-MS using MDPNA additive}

In order to investigate the effect of MDPNA at different concentrations of buffering salt, 15, 60, 250, and $500 \mathrm{mM}$ ammonium formate buffers were examined. When MDPNA was not added, all ion peaks of the seven peptides were observed at a concentration of $15 \mathrm{mM}$ ammonium formate (Fig. 2-1). However, with higher than $15 \mathrm{mM}$ ammonium formate, several peptide peaks disappeared due to signal suppression induced by the buffering salts (Figs. 2-2 through 2-4). The effect of MDPNA was reduced as the buffer concentrations increased, but MDPNA reduced signal suppression at all buffer concentrations examined. Although $\mathrm{N}$-Acetyl-Renin, ACTH 1-17, and ACTH 7-38 could not be detected, S/N ratios of Ang II, Ang I, [Glu1]-Fib, and ACTH
18-39 were improved at $500 \mathrm{mM}$ ammonium formate with MDPNA (Fig. 2-4, with MDPNA). All the ion peaks of the seven peptides were clearly observed at concentrations of 15,60 , and $250 \mathrm{mM}$ ammonium formate when MDPNA was added (Figs. 2-1 through 2-3). Buffering salt concentrations commonly used for LC range between 10 and $100 \mathrm{mM}$, except for ion-exchange chromatography. The range of buffer concentrations at which MDPNA was effective sufficiently covered this LC buffer concentration range. Moreover, MDPNA affects signal suppression caused by non-volatile salts $\left(50 \mathrm{mM} \mathrm{Na}_{2} \mathrm{PO}_{4}\right.$ and $\mathrm{NaCl}$ Figs. S-1 and S-2, supporting information; organic salts Fig. 1). Thus, various separation modes (e.g., gel filtration, HILIC, chromatofocusing, and reversed phase) can be applied to LC-MALDI-MS using MDPNA without special desalting steps.

\section{Effect of MDPNA at different $\mathrm{pH}$ levels}

Buffer $\mathrm{pH}$ is an important factor for optimizing chromatographic separation, and various $\mathrm{pH}$ conditions are used in LC. ${ }^{1,3,10,11)}$ The effect of adding MDPNA to the DHB matrix was further examined under different $\mathrm{pH}$ levels. Signal suppression was strong at $\mathrm{pH} 8.0$, but the $S / N$ ratio was significantly improved by adding MDPNA (Fig. 3-a). MDPNA also improved the $S / N$ ratios at $\mathrm{pH} 6.0$ and 4.0 (Figs. 3-b and 3-c). Similarly, the effect of adding MDPNA to the CHCA matrix was examined (Fig. S-8, supporting information). As in the DHB matrix, MDPNA reduced signal suppression 


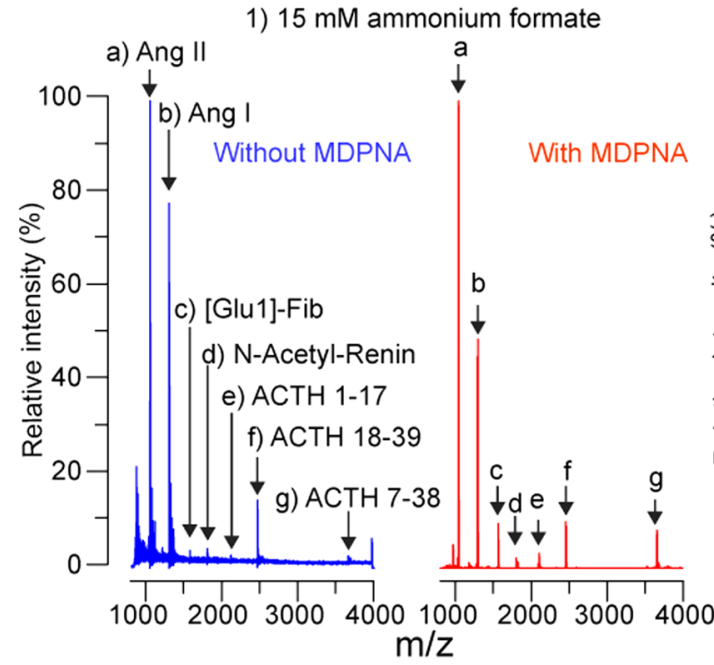

3) $250 \mathrm{mM}$ ammonium formate

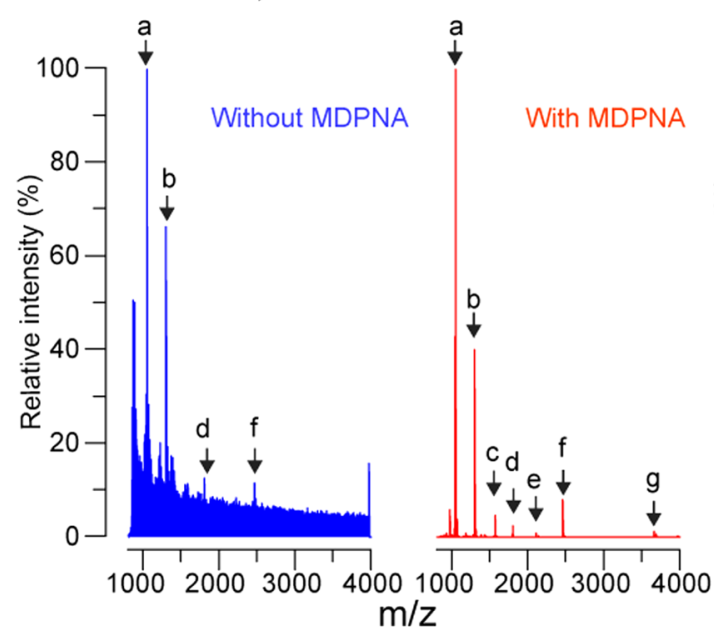

2) $60 \mathrm{mM}$ ammonium formate

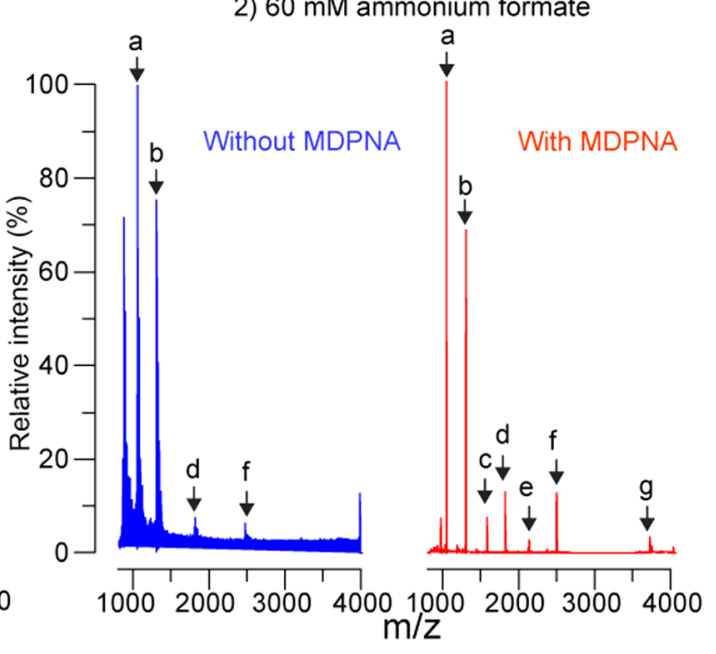

4) $500 \mathrm{mM}$ ammonium formate

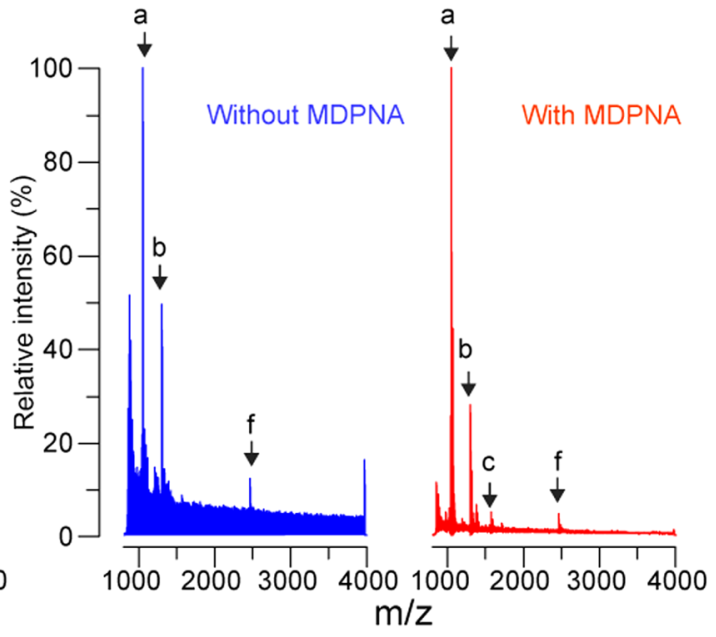

Fig. 2. Effect of MDPNA at different concentrations of ammonium formate buffer. The seven-peptide mix was dissolved in 15, 60, 250, or 500 mM ammonium formate buffer ( $\mathrm{pH}$ 5.0).

under all $\mathrm{pH}$ conditions. Thus, as a matrix additive for DHB and CHCA, MDPNA is applicable under all $\mathrm{pH}$ ranges used in LC.

Signal suppression was strong at $\mathrm{pH} 8.0$ amine buffer without MDPNA (Fig. 3-a, and Fig. S-8-a, supporting information), but it was not as strong as at low $\mathrm{pH}$ (Fig. 3-c, and Fig. S-8-c, supporting information). Low $\mathrm{pH}$ is important for sample-matrix co-crystallization processes. Approximately $0.1 \%(\mathrm{v} / \mathrm{v})$ TFA solution is widely used in MALDI sample preparation and the $\mathrm{pH}$ of $0.1 \%$ TFA solution is about 2 . The optimal $\mathrm{pH}$ for sample-matrix co-crystallization can be about 2. MDPNA and the other additives (EDPNA, PAC, and HEDP; Fig. S-5, PA; Fig. S-7) are relatively strong acids and the $\mathrm{p} K_{\mathrm{a}}$ values are near 2 (MDPNA: 1.43, EDPNA: 1.78, PAC: 1.74, HEDP: 1.97, and PA: 1.97). When the adequate additives were added to the samples containing buffering salts, the $\mathrm{pH}$ decreased to approximately 2 . The $\mathrm{pH}$-adjusting effect of the additives might be important for signal enhancement effects. The $\mathrm{p} K_{\mathrm{a}}$ of TFA is 0.05 and the $\mathrm{pH}$-adjusting effect cannot be expected. When a strong acid TFA was added as an additive, the $\mathrm{pH}$ was near 1 . TFA was not effective compared to MDPNA (Fig. S-7, supporting information). It has been reported that a matrix additive DAC reduced matrix clusters and chemical noises. However, DAC ( $\mathrm{pH} 4.5$ ) was ineffective to enhance salt tolerance (Fig. S-7).

\section{Effect of MDPNA on tryptic digests of proteins}

We have analyzed the effect of MDPNA on signal suppression using the seven peptides. The most common samples analyzed by MALDI-MS are tryptic-digested proteins, and the tryptic-digested samples contain various kinds of peptides. We confirmed the effect of MDPNA on signal suppression using multiple tryptic-digested samples. The BSA, ADH, ENO, and PhosB digests in 0.1\% TFA, pH 6.0 amine buffer, or $\mathrm{pH} 8.0$ amine buffer were measured with or without MDPNA using DHB as a matrix; the results are summarized in Fig. 4 (Mass spectra Fig. S-9, supporting information). The tryptic digests in $0.1 \%$ TFA (not containing buffering salt) with and without MDPNA exhibited marginal differences in sequence coverage ratios (Fig. 4, 0.1\% TFA). The sequence coverage ratios of the four tryptic digests in pH 6.0 and pH 8.0 amine buffers without MDPNA were significantly lower than that in $0.1 \%$ TFA (not containing buffering salt) without MDPNA because of signal suppression by amine buffering salts. However, the sequence coverage ratios of the tryptic digests in amine buffers were restored to the same level as under $0.1 \%$ TFA by adding MDPNA. Therefore, MDPNA effectively reduced the signal suppression of almost all the peptides by buffering salts. Similarly, the effect of adding MDPNA to the CHCA matrix was also examined (sequence coverage ratios Fig. S-10; mass spectra 
Matrix: DHB

a) $\mathrm{pH} 8.0$

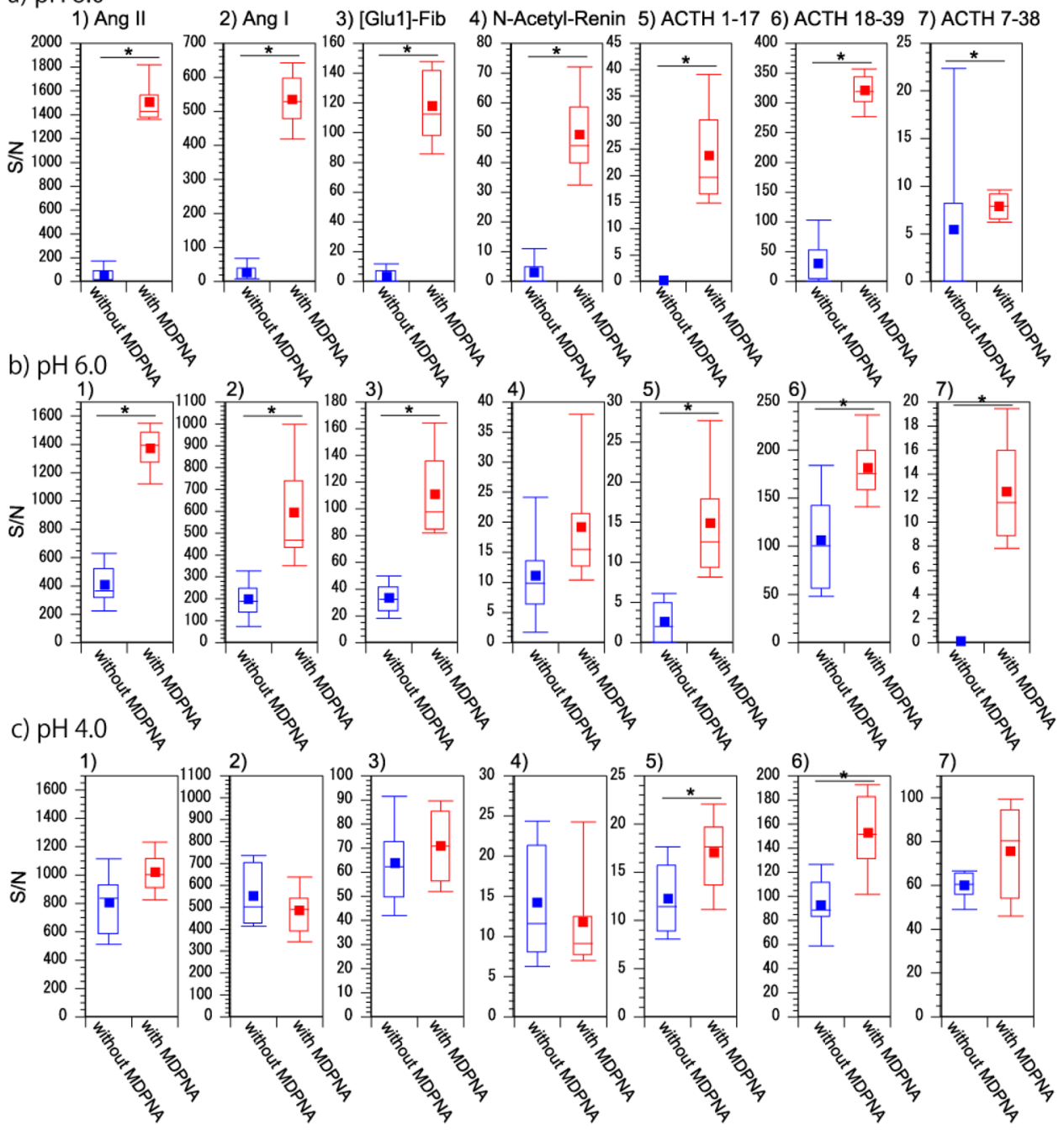

Fig. 3. Effect of adding MDPNA to the DHB matrix on ionization suppression by $\mathrm{pH} 8.0, \mathrm{pH} 6.0$, and $\mathrm{pH} 4.0$ amine buffers. Data for each peptide were analyzed by a Mann-Whitney $U$ test. Asterisks $\left({ }^{*} p<0.05\right)$ represent statistically significant differences. $(n=8)$.

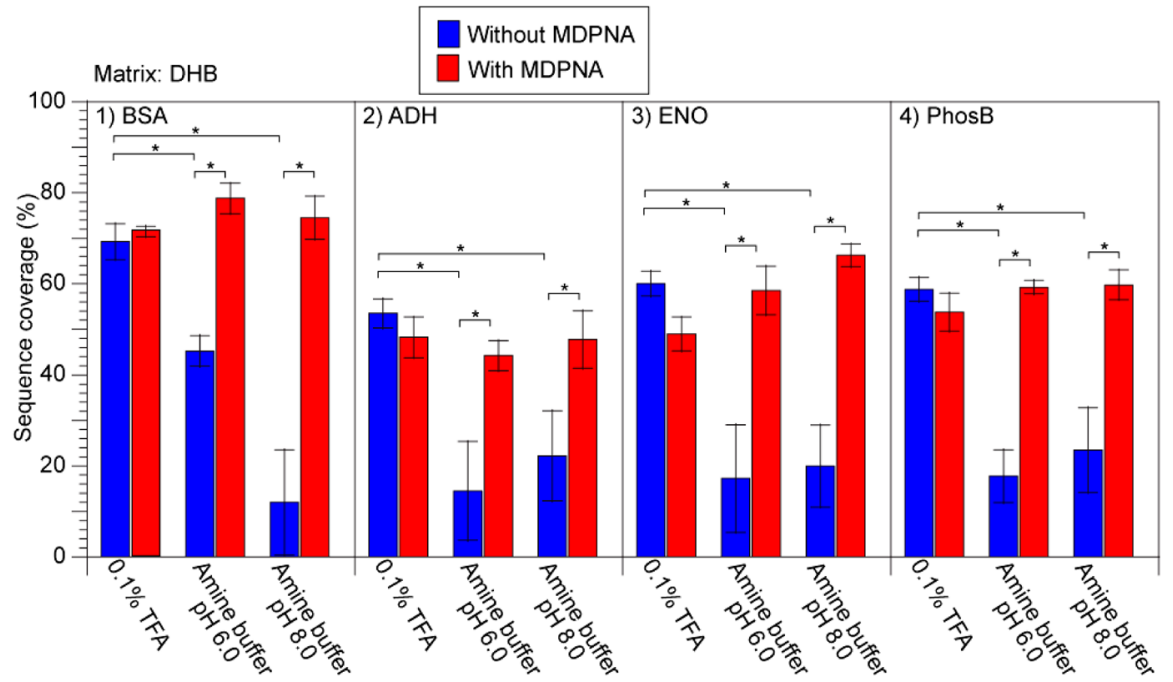

Fig. 4. Comparison of sequence coverage ratios with and without MDPNA after MALDI-MS peptide mass-fingerprinting analysis. DHB matrix was used. Data for each peptide were analyzed by a Kruskal-Wallis $H$ test followed by a Mann-Whitney $U$ test with Benjamini-Hochberg correction as a post-hoc test. Asterisks $\left({ }^{*} p<0.05\right)$ represent statistically significant differences. (means \pm S.D., $\left.n=6\right)$. 
Fig. S-11, supporting information). As in the DHB matrix, MDPNA effectively reduced the signal suppression of almost all the peptides by buffering salts.

\section{Zwitterionic HILIC-MALDI-MS}

Recently, zwitterionic HILIC has become a major separation mode because it is useful for hydrophilic compounds that cannot be retained on ODS. ${ }^{21)}$ However, zwitterionic HILIC requires salts in the mobile phase, which might hinder ionization in ion sources. Thus far, we have investigated the effect of the MDPNA additive. We applied it to actual LC-MALDI-MS using zwitterionic HILIC as a separation mode. A zwitterionic HILIC column, ZIC-HILIC, was used in this experiment. A $10 \mathrm{fmol}$ BSA digest sample was eluted by zwitterionic HILIC and analyzed by MALDI-MS. No peaks originating from the BSA digest sample were observed when MDPNA was not added (Figs. 5-1 and 5-2). However, such peaks were clearly observed when MDPNA was added (Figs. 5-1 and 5-2), and the detected peaks were subjected to MALDI-MS/MS analysis (Fig. S-12, supporting information). The results of LC-MALDI-MS/MS were analyzed by a Mascot MS/MS search, and BSA was hit with the top-score (score 96). MDPNA improved the sensitivity of HILIC-MALDI-MS with simple addition of an additive to the matrix without any special equipment or technique. The retention characteristics of zwitterionic HILIC reportedly change according to the $\mathrm{pH}$ and concentration of the

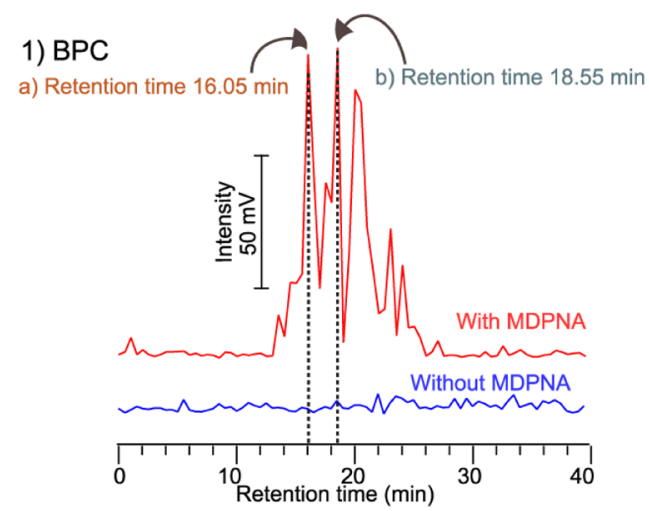

\section{2) Mass spectra}

a) Retention time: $16.05 \mathrm{~min}$

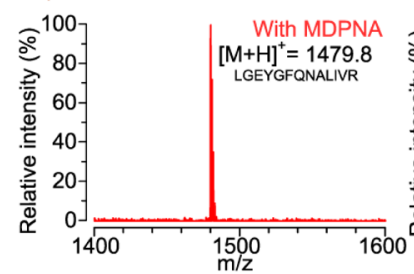

b) Retention time: $18.55 \mathrm{~min}$

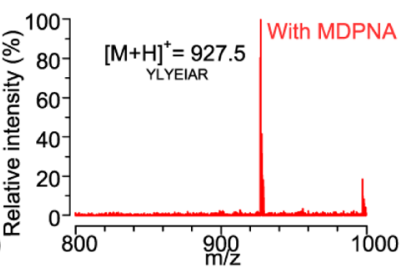

a) Retention time: 16.05 min
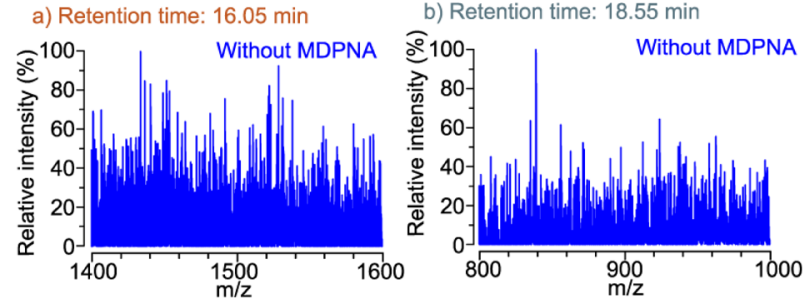

Fig. 5. (1) Base peak chromatograms (BPC) and (2) mass spectra of LC-MALDI-MS using HILIC as a separation mode. The mass spectra at retention times of 16.05 and $18.55 \mathrm{~min}$ are depicted. buffer. ${ }^{10,23)}$ For example, ZIC-HILIC exhibits better chromatographic resolution of peptides at high $\mathrm{pH}$ (7 to 8) and resembles separation on strong cation exchangers at low $\mathrm{pH}$ (3). ${ }^{11)}$ Peak tailing is more commonly observed in HILIC than in reversed-phase chromatography. The tailing peak can be sometimes improved by increasing the amount of buffering salts. ${ }^{11)}$ MDPNA is effective under a wide range of buffer concentrations and $\mathrm{pH}$ conditions. When MDPNA is used, the optimal mobile phase conditions for HILIC are freely selectable.

\section{Chromatofocusing-MALDI-MS}

MDPNA was applied to chromatofocusing-MALDI-MS. The chromatofocusing conditions were optimized based on Bates and Frey's method. ${ }^{22)}$ Buffers containing organic salts are generally avoided in LC-MALDI as well because they hinder the ionization of samples in MALDI. The sample was a mixture of two peptides, [Glu1]-Fib and ACTH 18-39, which have similar $\mathrm{p} I$ values. The chromatofocusing-MALDI-MS results are presented in Fig. 6. When MDPNA was not added to the DHB matrix, the extracted ion chromatogram peak heights of [Glu1]-Fib and ACTH 18-39 were low (Fig. 6-1). However, the peak heights were very high when MDPNA was added (Fig. 6-1). Based on retention times, the $\mathrm{p} I$ value of [Glu1]-Fib was estimated as 4.08 , and that of ACTH 18-39 was estimated as 3.9. The expected $\mathrm{p} I$ values calculated by computer simulation ${ }^{23,24)}$ were 4.09 for [Glu1]Fib and 4.00 for ACTH 18-39. The estimated $\mathrm{p} I$ values based on the retention times agreed to the $\mathrm{p} I$ values predicted by computer simulation. Figure 6-2 depicts the mass spectra of [Glu1]-Fib and ACTH 18-39. Their S/N ratios also improved when MDPNA was added. The peak intensities of [Glu1]Fib and ACTH 18-39 without MDPNA were weak, and we could not obtain sufficient MS/MS spectra (data not shown), but those of [Glu1]-Fib and ACTH 18-39 with MDPNA were sufficient to obtain good MS/MS spectra (Figs. S-13-a and S13-b, supporting information).

The retention time of chromatofocusing indicates the $\mathrm{pI}$ value of the analyte, and the mass spectrum yields structural information. The combination of MS and chromatofocusing can thus be useful for identifying unknown analytes. However, chromatofocusing requires buffers which generally avoided in MS analysis, and few studies have applied chromatofocusing as a separation mode in LC-MS because the mobile-phase salts commonly used in chromatofocusing suppress ionization. Shan et al. (2008) reported that chromatofocusing-MS was achieved using only buffers which are applicable to ESI-MS (Buffer A, $25 \mathrm{mM}$ ammonium bicarbonate and $25 \mathrm{mM}$ pyridine adjusted to $\mathrm{pH} 9.0$ with concentrated ammonium hydroxide; Buffer B, $25 \mathrm{mM}$ acetic acid and $25 \mathrm{mM}$ lactic acid), a binary pump gradient, and postcolumn addition of formic acid. ${ }^{25)}$ Their linear $\mathrm{pH}$ gradient was ideal, but the binary elution program for producing the ideal linear $\mathrm{pH}$ gradient was extremely complex. Nonetheless, a method for constructing a linear $\mathrm{pH}$ gradient using commonly used buffers in LC has been reported, ${ }^{22,26,27)}$ and the desired $\mathrm{pH}$ gradient is readily constructed (Fig. S-14, supporting information). Chromatofocusing-MS can become a practical analytical method when using MDPNA additive. 


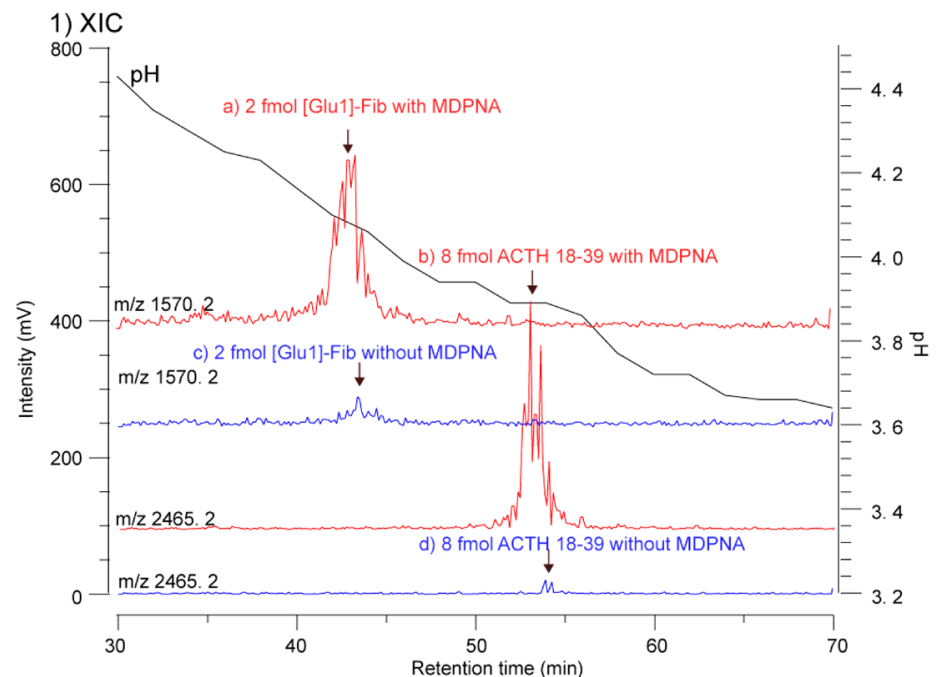

2) Mass spectra
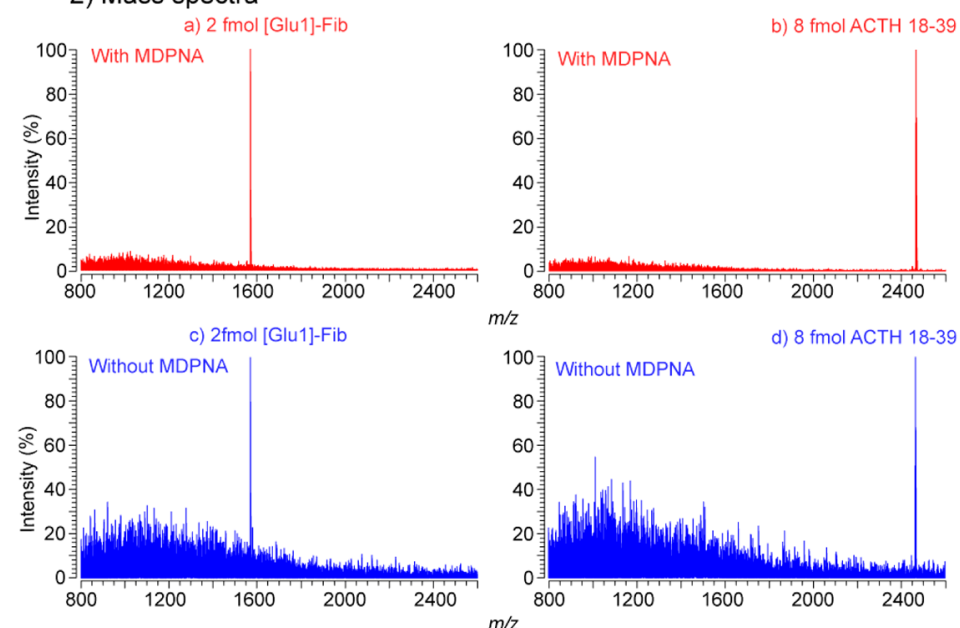

Fig. 6. (a) Extracted ion chromatogram (XIC) and (2) mass spectra after chromatofocusing-MALDI-MS. In (1), the XIC chromatograms and pH gradient are plotted.

\section{CONCLUSION}

We demonstrated that MALDI-MS using MDPNA as an additive is tolerant of the buffering salts commonly used in LC, and we achieved highly salt-tolerant LC-MALDIMS analysis using MDPNA. Although it is difficult to deal with extremely high concentrations (a buffer concentration range of several hundred $\mathrm{mM}$ ) simply by adding MDPNA, MDPNA affects various types of salts under a wide range of concentrations (15 to $250 \mathrm{mM}$ ammonium formate buffer) and $\mathrm{pH}$ conditions ( $\mathrm{pH} 4.0,6.0$, and 8.0). The acidification by MDPNA might be one reason of the effect of reducing signal suppression. This approach could overcome the limitations on LC conditions in LC-MALDI-MS analyses, and we can use the desired LC conditions in LC-MALDI-MS while maintaining high sensitivity.

\section{Acknowledgements}

This research was supported by the Japan Society for the Promotion of Science (JSPS) through the Funding Program for World-Leading Innovative R\&D on Science and Technology (FIRST) Program, initiated by the Council for Science and Technology Policy (CSTP).
The authors thank Dr. Hiroki Kuyama for helpful comments.

\section{REFERENCES}

1) M. C. Garcia, A. C. Hogenboom, H. Zappey, H. Irth. Effect of the mobile phase composition on the separation and detection of intact proteins by reversed-phase liquid chromatographyelectrospray mass spectrometry. J. Chromatogr. A 957: 187-199, 2002.

2) M. Liu, E. X. Chen, R. Ji, D. Semin. Stability-indicating hydrophilic interaction liquid chromatography method for highly polar and basic compounds. J. Chromatogr. A 1188: 255-263, 2008.

3) D. V. McCalley. Effect of buffer on peak shape of peptides in reversed-phase high performance liquid chromatography. J. Chromatogr. A 1038: 77-84, 2004.

4) L. Novakova. Challenges in the development of bioanalytical liquid chromatography-mass spectrometry method with emphasis on fast analysis. J. Chromatogr. A 1292: 25-37, 2013.

5) M. Holcapek, R. Jirasko, M. Lisa. Recent developments in liquid chromatography-mass spectrometry and related techniques. $J$. Chromatogr. A 1259: 3-15, 2012.

6) P. Donato, F. Cacciola, P. Q. Tranchida, P. Dugo, L. Mondello. Mass spectrometry detection in comprehensive liquid chromatography: Basic concepts, instrumental aspects, applications and trends. Mass Spectrom. Rev. 31: 523-559, 2012. 
7) R. Dams, T. Benijts, W. Gunther, W. Lambert, A. De Leenheer. Influence of the eluent composition on the ionization efficiency for morphine of pneumatically assisted electrospray, atmospheric-pressure chemical ionization and sonic spray. Rapid Commun. Mass Spectrom. 16: 1072-1077, 2002.

8) D. D. Frey, C. R. Narahari, R. C. Bates. Chromatofocusing. Encyclopedia of Life Sciences 1, 2001.

9) T. Ikegami, K. Tomomatsu, H. Takubo, K. Horie, N. Tanaka. Separation efficiencies in hydrophilic interaction chromatography. $J$. Chromatogr. A 1184: 474-503, 2008.

10) B. Buszewski, S. Noga. Hydrophilic interaction liquid chromatography (HILIC): A powerful separation technique. Anal. Bioanal. Chem. 402: 231-241, 2012.

11) P. Jandera. Stationary and mobile phases in hydrophilic interaction chromatography: A review. Anal. Chim. Acta 692: 1-25, 2011.

12) I. P. Smirnov, X. Zhu, T. Taylor, Y. Huang, P. Ross, I. A. Papayanopoulos, S. A. Martin, D. J. Pappin. Suppression of alphacyano-4-hydroxycinnamic acid matrix clusters and reduction of chemical noise in MALDI-TOF mass spectrometry. Anal. Chem. 76: 2958-2965, 2004

13) M. Asanuma, S. Fukuzawa, T. Matsuda, H. Hirota. The effect of sodium dodecyl sulfate and anion-exchange silica gel on matrixassisted laser desorption/ionization mass spectrometric analysis of proteins. Rapid Commun. Mass Spectrom. 23: 1647-1653, 2009.

14) H. Kuyama, K. Sonomura, O. Nishimura. Sensitive detection of phosphopeptides by matrix-assisted laser desorption/ionization mass spectrometry: Use of alkylphosphonic acids as matrix additives. Rapid Commun. Mass Spectrom. 22: 1109-1116, 2008.

15) mass++. http://www.first-ms3d.jp/english/achievement/software/ mass2.

16) R Development Core Team. http://www.R-project.org/ 2012.

17) S. Kjellstrom, O. N. Jensen. Phosphoric acid as a matrix additive for MALDI MS analysis of phosphopeptides and phosphopro- teins. Anal. Chem. 76: 5109-5117, 2004.

18) T. Moilanen, G. Scott, M. Newell, N. Garvie, M. A. Freeman. Bone scintigraphic appearance of asymptomatic hydroxyapatitecoated hip arthroplasties. J. Arthroplasty 12: 380-386, 1997.

19) T. Nishikaze, H. Okumura, H. Jinmei, J. Amano. Correlation between sweet spots of glycopeptides and polymorphism of the matrix crystal in MALDI samples. Mass Spectrom. (Tokyo) 1: A0006, 2012.

20) D. J. Harvey. Analysis of carbohydrates and glycoconjugates by matrix-assisted laser desorption/ionization mass spectrometry: An update for 2007-2008. Mass Spectrom. Rev. 31: 183-311, 2012.

21) P. J. Boersema, S. Mohammed, A. J. Heck. Hydrophilic inter action liquid chromatography (HILIC) in proteomics. Anal. Bioanal. Chem. 391: 151-159, 2008.

22) R. C. Bates, D. D. Frey. Quasi-linear $\mathrm{pH}$ gradients for chromatofocusing using simple buffer mixtures: Local equilibrium theory and experimental verification. J. Chromatogr. A 814: 43-54, 1998.

23) H. Li, A. D. Robertson, J. H. Jensen. Very fast empirical prediction and rationalization of protein $\mathrm{pK}_{\mathrm{a}}$ values. Proteins 61: $704-$ 721, 2005.

24) B. Neron, H. Menager, C. Maufrais, N. Joly, J. Maupetit, S. Letort, S. Carrere, P. Tuffery, C. Letondal. Mobyle: A new full web bioinformatics framework. Bioinformatics 25: 3005-3011, 2009.

25) L. Shan, J. A. Hribar, X. Zhou, D. J. Anderson. Gradient chromatofocusing-mass spectrometry: A new technique in protein analysis. J. Am. Soc. Mass Spectrom. 19: 1132-1137, 2008.

26) X. Kang, D. D. Frey. Chromatofocusing using micropellicular column packings with computer-aided design of the elution buffer composition. Anal. Chem. 74: 1038-1045, 2002.

27) X. Kang, R. C. Bates, D. D. Frey. High-performance chromatofocusing using linear and concave $\mathrm{pH}$ gradients formed with simple buffer mixtures II. Separation of proteins. J. Chromatogr. A 890: 37-43, 2000 